\title{
ON THE DIFFERENTIAL EQUATION OF A RAPIDLY ROTATING SLENDER ROD*
}

\author{
BY \\ W. D. LAKIN \\ University of Toronto
}

\begin{abstract}
In this work we consider a boundary-value problem arising from the transverse vibrations of a slender, finite, uniform rod which rotates with constant angular velocity about an axis through the rod's fixed end. The relevant dimensionless parameter is assumed to lie in a range corresponding to rapid rotation. The differential equation in this problem is fourth-order, linear, and takes its distinctive character from the simple turning point where the coefficient of the second derivative term vanishes. A significant feature is that the turning point is also a boundary point and hence outer expansions alone are not adequate for formation of a characteristic equation. Approximations valid at and away from the turning point are obtained and related through the method of matched asymptotic expansions. Outer expansions are required to be "complete" in the sense of Olver, and approximations are found for the Stokes multipliers which describe the analytic continuations of these expansions across Stokes lines in the complex plane. A consistent approximation to the characteristic equation is obtained, and the limiting behavior of the spectrum is derived analytically.
\end{abstract}

1. Introduction. In this paper we consider a boundary-value problem consisting of the fourth-order differential equation

$$
\phi^{\mathrm{iv}}-\frac{1}{2} a\left(1-y^{2}\right) \phi^{\prime \prime}+a y \phi^{\prime}-a \lambda \phi=0, \quad-1 \leq y \leq 0
$$

and the boundary conditions

$$
\phi(0)=\phi^{\prime}(0)=\phi^{\prime \prime}(-1)=\phi^{\prime \prime \prime}(-1)=0
$$

where $\phi=\phi(y)$ and $a$ is a dimensionless parameter assumed large, real, and positive, i.e.

$$
\text { ph } a=0 \text { and } a \gg 1 .
$$

The distinctive character of Eq. (1.1) comes from the simple turning point at $y=-1$ where the coefficient of $\phi^{\prime \prime}$ vanishes. A significant feature of the boundary-value problem is that this turning point is also an endpoint. Hence, a characteristic equation for the eigenvalue $\lambda$ cannot be formed using only approximations valid away from the turning point.

The reduced equation obtained by formally letting $a$ tend to infinity in (1.1) is of second order, and we are thus dealing with a singular perturbation problem. Inner approximations valid at and close to the turning point and outer approximations valid

* Received September 8, 1972; revised version received February 7, 1973. 
away from the turning point must be obtained separately and then related so that they asymptotically represent the same solutions (the central matching problem). We will use the method of matched asymptotic expansions. As usual, the variable $y$ will be assumed complex, and we consider bounded domains in the complex plane which contain the real interval $[-1,0]$. We also require that domains of validity are suitably restricted so that all outer expansions are complete in the sense of Olver [8]. Inner and outer approximations are matched using the asymptotic matching principle in the form stated by Fraenkel [3]. Approximations are also derived for the Stokes multipliers which describe the continuations of outer expansions across Stokes lines in the complex plane (the lateral connection problem). Using the matched approximations, we obtain a consistent approximation to the characteristic equation and derive analytically the limiting behavior of the spectrum as $a$ tends to infinity.

Eq. (1.1) is sometimes written in the modified form

$$
\phi^{\text {iv }}-\frac{1}{2} a\left(1-y^{2}\right) \phi^{\prime \prime}+a y \phi^{\prime}-\tilde{\lambda} \phi=0 .
$$

This equation is clearly appropriate for small $a$ and has also been used by Boyce, Di Prima, and Handelman [2] who, by means of minimum principles, have obtained bounds on $\bar{\lambda}$ for small to moderately large values of $a$. However, in the present asymptotic analysis for large $a$, this scaling of the eigenvalue is not consistent. The boundary conditions imply $\bar{\lambda}=O(a)$ which, in turn, leads to a breakdown in ordering in approximations to solutions of (1.4).

The boundary-value problem (1.1) and (1.2) arises in connection with the vibrations of a slender, uniform rod of length $l$ which rotates with constant angular velocity $\Omega$ about an axis through the rod's fixed end. We obtain Eq. (1.1) if the rod does not twist and vibrations are transverse to the plane of rotation. If $m$ is the linear mass density, $\omega$ the natural frequency of vibration, and $E I$ the bending stiffness of the rod, the parameter $a$ and eigenvalue $\lambda$ correspond to the ratios (see [1])

$$
a=\frac{m l^{4} \Omega^{2}}{E I} \text { and } \lambda=(\omega / \Omega)^{2} .
$$

Rapid rotation thus leads to large values of $a$. A frequency transformation derived by Lo and Renbarger [6] may be used to reduce vibrations not transverse to the plane of rotation to the present case. Recent interest in rotating rods stems from the effects of flexible appendages on the overall stability of "spin-stabilized" space satellites. The "pure spin" satellite reference motion of Hughes and Fung [4] is a solution of (1.1) and (1.2).

The second- and fourth-derivative terms in (1.1) and the Orr-Sommerfeld equation of hydrodynamic stability are quite similar, especially when the basic velocity profile is plane Poiseuille flow. Indeed, the class of special functions we use to obtain inner approximations has been developed by Reid [9] to study the Orr-Sommerfeld equation. Beyond this initial similarity, however, there are significant differences. The firstderivative term in (1.1) plays an important role at lowest order in all approximations. Because of this, the first inner approximation to a partially balanced solution of (1.1) requires the full generality of the special functions. The boundary conditions (1.2) also differ from the conditions associated with the stability of parallel flows. We require second and third derivatives at the turning point itself and the boundary point $y=0$ lies directly on a Stokes line in the complex plane. 
In what follows, a $D$ or prime will always denote differentiation with respect to the argument of the function involved. This work has been partially supported by the National Research Council of Canada under grant number A7850.

2. Transformation to standard form. A preliminary transformation of Eq. (1.1) will first be made which explicitly brings out the turning point nature of the problem. We define the Langer variable $\eta$ by

$$
\eta=\frac{1}{2}\left[3 \int_{-1}^{\nu}\left(1-y^{2}\right)^{1 / 2} d y\right]^{2 / 3} .
$$

Then, $\eta(y)$ is analytic at $y=-1$ and has the expansion

$$
\eta(y)=(y+1)-\frac{1}{10}(y+1)^{2}-\frac{2}{175}(y+1)^{3}+\cdots .
$$

Thus, bounded domains in the $y$-plane containing the turning point $y=-1$ are mapped by relation (2.1) on bounded domains in the $\eta$-plane containing the origin. We next define a new dependent variable $\chi(\eta)$ by

$$
\chi(\eta)=\phi(y) .
$$

Under this change of both independent and dependent variables, Eq. (1.1) becomes the required standard form

$$
\epsilon^{3} \chi^{\text {iv }}+6 \epsilon^{3} \gamma \chi^{\prime \prime \prime}-\left(\eta+\epsilon^{3} f_{1}\right) \chi^{\prime \prime}-\left(h_{0}+\epsilon^{3} h_{1}\right) \chi^{\prime}-\lambda \eta^{\prime-4} \chi=0
$$

where

$$
\epsilon=a^{-1 / 3}, \text { ph } \epsilon=0, \quad \epsilon \ll 1,
$$

and $\gamma, f_{1}, h_{0}$, and $h_{1}$ are all analytic functions of $\eta$ in bounded domains containing $\eta=0$. The function $\gamma(\eta)$ is defined by the relation

$$
\gamma(\eta)=\eta^{\prime \prime} / \eta^{\prime 2}
$$

and near $\eta=0$ it has the expansion

$$
\gamma(\eta)=\gamma_{0}+\gamma_{0}^{\prime} \eta+\cdots, \quad \gamma_{0}=-\frac{1}{5} \text { and } \gamma_{0}^{\prime}=-\frac{26}{175} .
$$

In terms of $\gamma$, the remaining coefficients in Eq. (2.4) are

$$
h_{0}(\eta)=1+3 \gamma \eta, \quad h_{1}(\eta)=-\gamma^{\prime \prime}-7 \gamma \gamma^{\prime}-6 \gamma^{3}, \quad f_{1}(\eta)=-4 \gamma^{\prime}-11 \gamma^{2} .
$$

Eq. (2.4) is not in normal form since a third derivative appears, but this does not cause any difficulties.

Approximations to solutions of Eq. (2.4) exhibit certain symmetries in the complex $\eta$-plane. To take full advantage of this fact, we will seek asymptotic expansions for seven exact solutions. While the forms of these exact solutions are, of course, unknown, the solutions may be specified by their asymptotic properties. Thus, to within a multiplicative constant, we may uniquely define four solutions as follows:

(i) The solution $U_{0}(\eta)$ is well balanced in bounded domains containing the turning point $\eta=0$. 
(ii) The three solutions $V_{k}(\eta)$ are recessive in the sectors $S_{k}$ bounded by anti-Stokes lines $\left(k=1,2,3\right.$; see Fig. 1). To within multiplicative constants and multiples of $U_{0}$, we may also uniquely define three additional exact solutions $U_{k}(\eta)$ by the properties:

(iii) The solutions $U_{k}(\eta)$ are purely balanced in the sectors $T_{k}$ bounded by Stokes lines $(k=1,2,3$; see Fig. 1$)$.

With the exception of $U_{0}(\eta)$, these solutions, or more precisely their outer expansions, exhibit the Stokes phenomenon. Since the equation is only fourth order, the seven exact solutions must be related through three exact (but unknown) connection formulas. One aim of this work is to derive approximations to these exact relations and hence obtain approximations to the Stokes multipliers which specify the required analytic continuations of the outer expansions.

3. The inner expansions. In this section, we wish to derive (inner) approximations valid close to and at the turning point: Balancing the first, second, and fourth derivative terms in (2.4) shows that the critical layer at $\eta=0$ will have thickness $O(\epsilon)$. Accordingly, we define the inner variable $\xi$ by

$$
\xi=\eta / \epsilon
$$

and let

$$
\tilde{\chi}(\xi)=\chi(\eta) .
$$

With this scaling, the relevant form of (2.4) near the turning point now becomes

$\tilde{\chi}^{i \nabla}+6 \epsilon \gamma(\epsilon \xi) \tilde{\chi}^{\prime \prime \prime}-\left[\xi+\epsilon^{2} f_{1}(\epsilon \xi)\right] \tilde{\chi}^{\prime \prime}-\left[1+3 \epsilon \xi \gamma(\epsilon \xi)+\epsilon^{3} h_{1}(\epsilon \xi)\right] \tilde{\chi}^{\prime}-\epsilon \lambda e^{-4}(\epsilon \xi) \tilde{\chi}=0$

where the function

$$
e(\eta)=1-\frac{1}{5} \eta-\frac{19}{350} \eta^{2}+\cdots
$$

denotes $\eta^{\prime}$ re-expressed in terms of $\eta$. Eq. (3.3) suggests inner expansions of the form

$$
\tilde{\chi}=\sum_{n=0}^{\infty} \tilde{\chi}^{(n)}(\xi) \epsilon^{n} .
$$

Using this relation, expanding the coefficients in (3.3), and equating powers of $\epsilon$ to zero, we now obtain a sequence of differential equations for the $\tilde{\chi}^{(n)}$. To write these equations

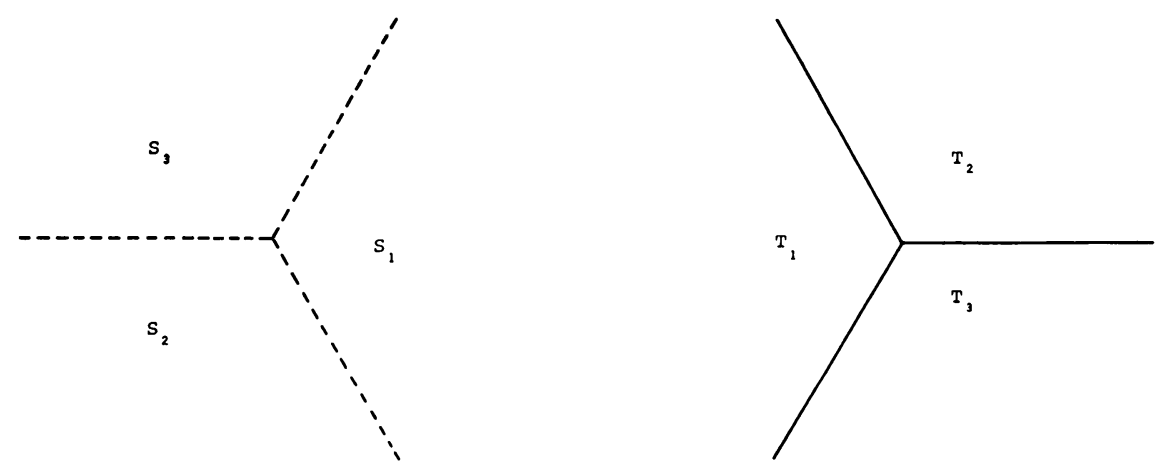

Fig. 1. The anti-Stokes lines (left) and the Stokes lines (right) in the $\eta$-plane. 
in compact form, we let

$$
D=d / d \xi, \quad A=D^{2}-\xi, \quad B=D^{2}+A .
$$

Then

$$
\begin{aligned}
& D A D \tilde{\chi}^{(0)}=0 \\
& D A D \tilde{\chi}^{(1)}=I_{1} \tilde{\chi}^{(0)}, \\
& D A D \tilde{\chi}^{(2)}=I_{1} \tilde{\chi}^{(1)}+I_{2} \tilde{\chi}^{(0)},
\end{aligned}
$$

etc., where $I_{1}$ and $I_{2}$ are the operators

$$
\begin{aligned}
& I_{1}=-3 \gamma_{0} B D+\lambda, \\
& I_{2}=-\left[\xi\left(3 \gamma_{0}^{\prime} B D-\frac{4}{5} \lambda\right)+\left(11 \gamma_{0}^{2}+4 \gamma_{0}^{\prime}\right) D^{2}\right] .
\end{aligned}
$$

We wish to obtain partial sums for seven inner expansions which will be denoted by $\tilde{u}_{0}(\xi), \tilde{u}_{k}(\xi)$, and $\tilde{v}_{k}(\xi)(k=1,2,3)$. These expansions are defined by the requirement that as $|\xi| \rightarrow+\infty$ they have same asymptotic behavior as the seven exact solutions $U_{0}, U_{k}$, and $V_{k}$, respectively. Thus, $\tilde{u}_{0}$ must be well balanced, $\tilde{u}_{k}$ must be balanced in $T_{k}$, and $\tilde{v}_{k}$ must be recessive in $S_{k}$. These conditions uniquely specify $\tilde{u}_{0}$ and $\tilde{v}_{k}$ to within a multiplicative constant. However, care must be taken with the $\tilde{u}_{k}$ since, in addition to the normalization factors, arbitrary multiples of $\tilde{u}_{0}$ may be added to these expansions without changing their asymptotic properties.

The eigenvalue $\lambda$ first appears in (3.5) at order $\epsilon$ and, in fact, to obtain a consistent first approximation to $U_{0}^{\prime \prime \prime}(0)$ one must go to order $\epsilon^{3}$. A large number of particular solutions for inhomogeneous fourth-order equations are thus required. Fortunately, a class of special functions developed to study solutions of the Orr-Sommerfeld equation can also be used in the present problem. These generalized Airy functions are discussed by Reid [9] and we therefore list below only definitions and relevant properties.

The functions $A_{k}(\xi ; p, q)$ and $B_{k}(\xi ; p, q)(k=1,2,3)$. If $C_{k}$ denotes either $A_{k}$ or $B_{k}$, these special functions are defined by the contour integrals

$$
C_{k}(\xi ; p, q)=\frac{1}{2 \pi i} \int t^{-p} \log _{a} t \exp \left[\xi t-t^{3} / 3\right] d t
$$

where the paths of integration $\mathfrak{L}_{k}$ for $A_{k}$ and $\odot_{k}$ for $B_{k}$ are as in Fig. 2. When $q=0$, we write

$$
A_{k}(\xi ; p, 0)=A_{k}(\xi, p)
$$

In particular, $A_{k}(\xi, 0)$ is the usual Airy function. Similarly, when $q=0$ the paths of integration $P_{k}$ can be closed to give the path $\odot_{0}$ which is a circle centered at $t=0$. In this case, we write

$$
B_{k}(\xi ; p, 0)=B_{0}(\xi, p) .
$$

Using residues, one easily obtains

$$
\begin{aligned}
B_{0}(\xi, p) & =0, & & p \leq 0, \\
& =b_{p}(\xi), & & p=1,2,3, \cdots,
\end{aligned}
$$

where $b_{p}(\xi)$ is a polynomial of degree $p-1$ and 

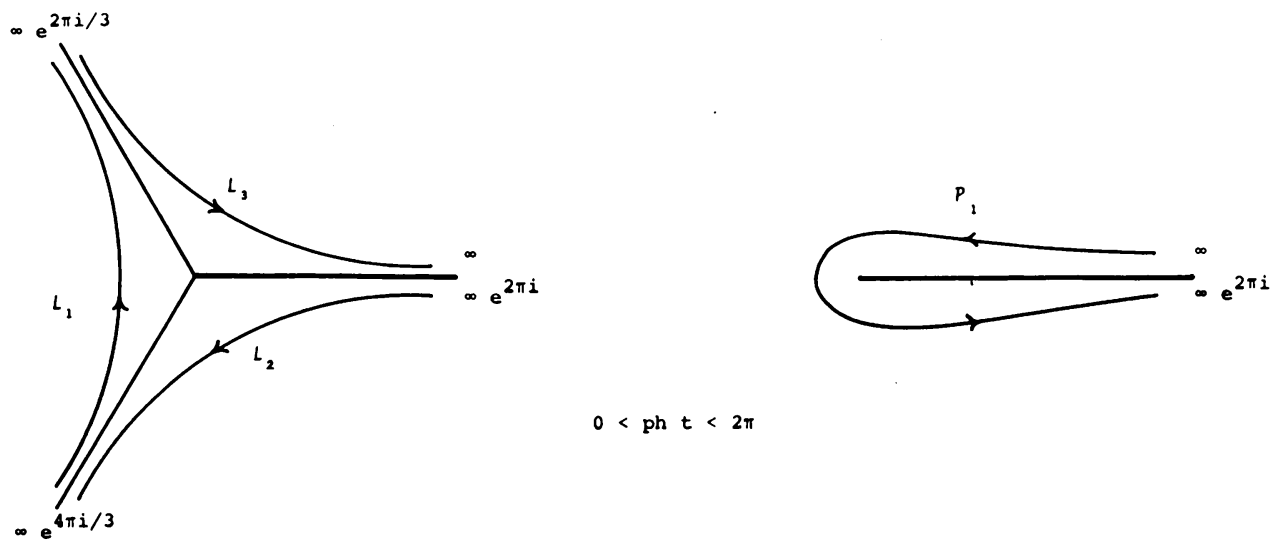

Fig. 2. The paths of integration $\mathfrak{L}_{k}$ (left) and $\mathscr{P}_{1}$ (right) in the complex $t$-plane for the functions $A_{k}(\xi ; p, q)$. and $B_{1}(\xi ; p, q)$ respectively $(k=1,2,3)$. For index $q=0$, the branch cut along the positive real axis is unnecessary and the paths $\boldsymbol{P}_{k}$ may be closed to form the circular path $\boldsymbol{P}_{0}$.

$$
b_{1}(\xi)=1, \quad b_{2}(\xi)=\xi, \quad b_{3}(\xi)=\frac{\xi^{2}}{2 !}, \quad b_{4}(\xi)=\frac{\xi^{3}}{3 !}-\frac{1}{3}, \quad \text { etc. }
$$

Derivatives of $C_{k}(\xi ; p, q)$ are given by the simple relation

$$
D^{m} C_{k}(\xi ; p, q)=C_{k}(\xi ; p-m, q) .
$$

Similarly,

$$
C_{k}(\xi ; p, q+1)=-\frac{\partial}{\partial p} C_{k}(\xi ; p, q) .
$$

If so desired, the recursion formula

$C_{k}(\xi ; p-3, q)-\xi C_{k}(\xi ; p-1, q)+(p-1) C_{k}(\xi ; p, q)-q C_{k}(\xi ; p, q-1)=0$

makes it sufficient to restrict $p$ to the range $-1 \leq p \leq 1$.

By using Cauchy's theorem, exact connection formulas relating the various functions can be obtained. For $q=1$, these have the form

$$
\begin{aligned}
& B_{3}(\xi ; p, 1)-B_{1}(\xi ; p, 1)=2 \pi i A_{2}(\xi, p) \\
& B_{1}(\xi ; p, 1)-B_{2}(\xi ; p, 1)=2 \pi i A_{3}(\xi, p) \\
& B_{2}(\xi ; p, 1)-B_{3}(\xi ; p, 1)=2 \pi i\left[A_{1}(\xi, p)+B_{0}(\xi, p)\right]
\end{aligned}
$$

A further general relation is

$$
\sum_{k=1}^{3} A_{k}(\xi ; p, q)=-B_{1}(\xi ; p, q) .
$$

The asymptotic properties of these generalized Airy functions are similar to the properties defining the exact solutions of equation (2.4). As $|\xi| \rightarrow+\infty, B_{0}(\xi, p)$ is well balanced, $B_{k}(\xi ; p, q)$ with $q \neq 0$ is balanced in the sector $T_{k}$, while $A_{k}(\xi ; p, q)$ is recessive in the sector $S_{k}$. In particular, for $\xi \in T_{2} \cup T_{3}, A_{1}(\xi, p)$ has the expansion 


$$
\begin{aligned}
A_{1}(\xi, p) \sim \frac{1}{2} \pi^{-1 / 2} \exp (-p \pi i) \xi^{-(2 p+1) / 4} \exp (-\zeta) \sum_{m=0}^{\infty}(-1)^{m} a_{m}(p) \zeta^{-m} \\
\quad\left(-\frac{2 \pi}{3}<p h \xi<\frac{2 \pi}{3}\right)
\end{aligned}
$$

with $\zeta=\frac{2}{3} \xi^{3 / 2}, a_{0}(p)=1, a_{1}(p)=\frac{1}{72}\left(12 p^{2}+24 p+5\right)$, etc. For $\xi \in T_{1}, B_{1}(\xi ; 1,1)$ has the expansion

$$
B_{1}(\xi ; 1,1) \sim-\log \xi-\gamma+\sum_{m=1}^{\infty} \frac{(3 m-1) !}{3^{m} m !} \xi^{-3 m} \quad\left(-\frac{2 \pi}{3}<p h \xi<0\right)
$$

where $\gamma$ here is the Euler constant. Expansions for different values of $p$ and $q$ may be obtained using (3.16) and (3.17), while $C_{2}$ and $C_{3}$ may be obtained from $C_{1}$ by replacing $\xi$ in $(3.11)$ by $\xi \exp [ \pm 2 \pi i / 3]$.

The generalized Airy functions are solutions of the differential equation

$$
(A D+p-1) C_{k}(\xi ; p, q)=q C_{k}(\xi ; p, q-1) .
$$

Since $C_{k}(\xi ; p, q)=D C_{k}(\xi ; p+1, q)$ and $D A D=(A D-1) D$, Eq. (3.23) thus leads to the differential equation

$$
D A D C_{k}(\xi ; p+1, q)=-p C_{k}(\xi ; p, q)+q C_{k}(\xi ; p, q-1)
$$

required in the present problem.

The expansion $\tilde{u}_{0}(\xi)$. Consider now the inner expansion $\tilde{u}_{0}$ which must be well balanced as $|\xi| \rightarrow+\infty$. Eqs. (3.7), (3.24), and the required asymptotic behavior give that $\tilde{u}_{0}{ }^{(0)}$ must be a multiple of $B_{0}(\xi, 1)$. If we set the normalization constant in $\tilde{u}_{0}{ }^{(0)}$ to unity and require that for $n \geq 1 \tilde{u}_{0}{ }^{(n)}$ contains no multiple of $\tilde{u}_{0}{ }^{(0)}$, then

$$
\begin{aligned}
& \tilde{u}^{\left({ }^{(0)}\right.}(\xi)=B_{0}(\xi, 1)=1, \\
& \tilde{u}_{0}^{(1)}(\xi)=-\lambda \xi, \\
& \tilde{u}_{0}^{\left({ }^{(2)}\right.}(\xi)=\frac{1}{4}\left[\lambda^{2}-\frac{7}{5} \lambda\right] \xi^{2},
\end{aligned}
$$

etc. Thus, in terms of the outer variable $\eta=\epsilon \xi, \tilde{u}_{0}$ has the form

$$
\tilde{u}_{0}=\left[1-\lambda \eta+\frac{1}{4}\left(\lambda^{2}-\frac{7}{5} \lambda\right) \eta^{2}+\cdots\right]+O\left(\epsilon^{3}\right) .
$$

The expansions $\tilde{v}_{k}(\xi)(k=1,2,3)$. These inner expansions are uniquely defined up to multiplicative constants by the requirement that as $|\xi| \rightarrow+\infty \quad \tilde{v}_{k}$ must be recessive in the sector $S_{k}$. Thus, $\tilde{v}_{k}{ }^{(0)}$ must be a multiple of $A_{k}(\xi ; 1)$. Setting the normalization constant to unity and requiring that for $n \geq 1{\tilde{v}_{k}}^{(n)}$ should contain no multiple of $\tilde{v}_{k}{ }^{(0)}$, we easily obtain

and

$$
\begin{aligned}
& \tilde{v}_{k}{ }^{(0)}=A_{k}(\xi, 1) \\
& \tilde{v}_{k}{ }^{(1)}=-\frac{3}{2} \gamma_{0} A_{k}(\xi,-1)-\lambda A_{k}(\xi, 2),
\end{aligned}
$$

$$
\begin{aligned}
\tilde{v}_{k}^{(2)}= & \frac{3}{4}\left[\frac{3}{2} \gamma_{0}{ }^{2}-\gamma_{0}{ }^{\prime}\right] A_{k}(\xi,-3) \\
& +\left[\frac{4}{5} \lambda+\frac{3}{2} \gamma_{0} \lambda-2 \gamma_{0}{ }^{2}+2 \gamma_{0}{ }^{\prime}\right] A_{k}(\xi, 0) \\
& +\frac{\lambda}{2}\left[\lambda+3 \gamma_{0}-\frac{4}{5}\right] A_{k}(\xi, 3) .
\end{aligned}
$$


Since $A_{k}(\xi, 0)$ will appear in the inhomogeneous portion of the equation for $\tilde{v}_{k}^{(3)}$, a term involving $A_{k}(\xi ; 1,1)$ is present in $\tilde{v}_{k}$ at order $\epsilon^{3}$.

The expansions $\tilde{u}_{k}(\xi)(k=1,2,3)$. As $|\xi| \rightarrow+\infty$ the three inner expansions $\tilde{u}_{k}$ must be balanced in the sectors $T_{k}$, and to obtain even the first approximations $\tilde{u}_{k}{ }^{(0)}$ a nonzero index $q$ is necessary. The asymptotic behavior implies that $\tilde{u}_{k}{ }^{(0)}$ must involve a multiple of $B_{k}(\xi ; 1,1)$. However, care must be taken since adding an arbitrary multiple of $\tilde{u}_{0}{ }^{(0)}$ will not alter the asymptotic behavior. Thus, $\tilde{u}_{k}{ }^{(0)}$ must be of the form

$$
\tilde{u}_{k}{ }^{(0)}(\xi)=c_{k} B_{k}(\xi ; 1,1)+b_{k} B_{0}(\xi, 1)
$$

where $b_{k}$ and $c_{k}$ are normalization constants. If we require that $\tilde{u}_{k}{ }^{(0)}$ should contain no multiple of $\tilde{u}_{0}{ }^{(0)}$, then we must choose $b_{k} \equiv 0$. However, this does not imply that for $n \geq 1$ the higher approximations $\tilde{u}_{k}{ }^{(n)}$ will not contain multiples of $B_{0}(\xi, p)$ with $p>1$. Indeed, $\tilde{u}_{k}{ }^{(n)}$ will in general contain a well-balanced term in addition to the portion involving $B_{k}(\xi ; p, q)$ with $q$ nonzero. To complete the normalization of these expansions, we set $c_{k}=1$ and require that for $n \geq 1 \tilde{u}_{k}{ }^{(n)}$ should contain no multiple of $\tilde{u}_{k}{ }^{(0)}$. We now find

$$
\begin{gathered}
\tilde{u}_{k}{ }^{(0)}(\xi)=B_{k}(\xi ; 1,1), \\
\tilde{u}_{k}^{(1)}(\xi)^{*}=-\lambda B_{k}(\xi ; 2,1)-\frac{3}{2} \gamma_{0} B_{k}(\xi ;-1,1)+\left(3 \gamma_{0}-\lambda\right) \xi, \\
\tilde{u}_{k}^{(2)}(\xi)=\frac{\lambda}{2}\left[\lambda+3 \gamma_{0}-\frac{4}{5}\right] B_{k}(\xi ; 3,1)+\frac{3}{4}\left[\frac{3}{2}{\gamma_{0}}^{2}-\gamma_{0}{ }^{\prime}\right] B_{k}(\xi ;-3,1) \\
+\left[\lambda\left(\frac{3}{2} \gamma_{0}+\frac{4}{5}\right)-2{\gamma_{0}}^{2}+2 \gamma_{0}{ }^{\prime}\right] B_{k}(\xi ; 0,1) \\
+\frac{1}{8}\left[3 \lambda^{2}-\lambda\left(3 \gamma_{0}-\frac{4}{5}\right)-18 \gamma_{0}{ }^{2}+6 \gamma_{0}{ }^{\prime}\right] \xi^{2},
\end{gathered}
$$

etc. For $n \geq 1$, the expressions for $\tilde{u}_{k}^{(3 n)}, \tilde{u}_{k}^{(3 n+1)}$, and $\tilde{u}_{k}^{(3 n+2)}$ will in general involve terms containing $B_{k}(\xi ; p, q)$ with $q=0,1, \cdots, n+1$.

4. The outer expansions. We now wish to derive seven outer expansions valid away from the turning point. These expansions will be denoted by $\bar{u}_{0}(\eta), \bar{u}_{k}(\eta)$, and $\bar{v}_{k}(\eta)(k=1,2,3)$ and are defined by the conditions that $\bar{u}_{0}$ is well balanced, $\bar{u}_{k}$ is balanced in $T_{k}$, while $\bar{v}_{k}$ is recessive in $S_{k}$. We will also require that all of these expansions must be "complete" in the sense of Olver [8]. Basically, this implies that different asymptotic representations of a given solution must have their domains of validity restricted to nonoverlapping sectors bounded by Stokes lines (although, in the usual Poincaré sense, the expressions remain valid in larger overlapping regions). The importance of using complete expansions for fourth-order equations has been shown by Lakin and Reid [5] in a study of the Orr-Sommerfeld equation.

The expansions $\bar{u}_{0}(\eta)$ and $\bar{u}_{k}(\eta)$. To obtain the well-balanced and partially-balanced outer expansions, we will assume a formal expansion $\bar{\chi}(\eta)$ of the form

$$
\bar{\chi}=\sum_{n=0}^{\infty} \bar{\chi}^{(n)}(\eta) \epsilon^{3 n}
$$

Substituting (4.1) into (2.4) and equating powers of $\epsilon^{3}$ to zero now gives the sequence of relations

$$
\mathscr{L} \bar{\chi}^{(0)}=0
$$


and

$$
\mathfrak{L} \bar{\chi}^{(n+1)}=\mathfrak{M} \bar{\chi}^{(n)}, \quad n \geq 0,
$$

with

$$
\mathscr{L}=\eta \frac{d^{2}}{d \eta^{2}}+[1+3 \eta \gamma(\eta)] \frac{d}{d \eta}+\lambda \eta^{\prime-4}
$$

and

$$
\mathscr{T}=\frac{d^{4}}{d \eta^{4}}+6 \gamma(\eta) \frac{d^{3}}{d \eta^{3}}-f_{1}(\eta) \frac{d^{2}}{d \eta^{2}}-h_{1}(\eta) \frac{d}{d \eta} .
$$

Power-series solutions of (4.2) may be obtained by the method of Frobenius. One solution is analytic at $\eta=0$ and thus corresponds to a multiple of $\bar{u}_{0}{ }^{(0)}$. If we normalize so that $\bar{u}_{0}^{(0)}(0)=1$, then

$$
\bar{u}_{0}^{(0)}(\eta)=1-\lambda \eta+\frac{\lambda}{4}\left(\lambda-\frac{7}{5}\right) \eta^{2}+O\left(\eta^{3}\right) .
$$

Note that this is simply the lowest-order term in $\tilde{u}_{0}(\xi)$ expressed in terms of the outer variable $\eta$ (see. (3.26)). For $n \geq 1, \bar{u}_{0}{ }^{(n)}$ will contain no constant term and hence $\bar{u}_{0}^{(n)}(0)=0$.

Let $\bar{w}(\eta)$ denote an expansion of the form (4.1) such that $\bar{w}^{(0)}$ is the second Frobeniustype solution of (4.2). This solution has a logarithmic branch point at the origin and is of the form

$$
\bar{w}^{(0)}(\eta)=P(\eta)+\bar{u}_{0}^{(0)}(\eta) \log \eta
$$

where $P(\eta)$ is a series in powers of $\eta$ and $P(0) \neq 0$. To facilitate matching to the inner expansions, we will choose $P(0)=\gamma$, the Euler constant. With this normalization,

$$
\begin{aligned}
P(\eta)=\gamma+\left[\frac{3}{5}+\lambda(2-\gamma)\right] \eta & +\left[3 \gamma_{0}^{\prime}-\frac{9}{25}+\frac{7 \lambda}{20}\left(\gamma-\frac{1}{2}\right)-\frac{\lambda^{2}}{4}(\gamma-3)\right] \eta^{2} \\
+ & O\left(\eta^{3}\right) .
\end{aligned}
$$

To fix the branch in (4.7), it is convenient to place a cut along the negative real $\eta$-axis and consider ph $\eta$ in the range

$$
-\pi<\operatorname{ph} \eta<\pi \text {. }
$$

However, a single exact solution of (2.4) cannot be asymptotic to $\bar{w}$ for all ph $\eta$ in this range. In the complete sense, $\bar{w}$ is a valid asymptotic representation for a given exact solution of balanced type only in a sector of angle $2 \pi / 3$ bounded by Stokes lines. We thus define the three outer expansions $\bar{u}_{k}$ by the relations

$$
\begin{aligned}
& \bar{u}_{2}(\eta)=\bar{w}(\eta) \text { for } \quad \eta \in T_{2}, \\
& \bar{u}_{3}(\eta)=\bar{w}(\eta) \text { for } \quad \eta \in T_{3}, \\
& \bar{u}_{1}(\eta)=\bar{w}(\eta) \text { for } \quad \eta \in S_{2} \cap T_{1} .
\end{aligned}
$$

On crossing the branch cut, $\bar{u}_{1}(\eta)$ may change its form but not its value. Thus, to define $\bar{u}_{1}$ in the remainder of $T_{1}$, we must take into account the multiples of $2 \pi i$ which are 
introduced by the logarithmic portions in $\bar{w}^{(n)}$ for $n \geq 0$ (see (4.7)). In particular, since $\bar{u}_{0}{ }^{(0)}(0)=1$, we must have

$$
\bar{u}_{1}(\eta)=\bar{w}(\eta)+2 \pi i+O\left(\epsilon^{3}\right) \text { for } \eta \in S_{3} \cap T_{1} .
$$

The equation $\mathscr{L} \bar{\chi}^{(0)}=0$ is simply a transformed version of the reduced equation

$$
-\frac{1}{2}\left(1-y^{2}\right) \phi^{\prime \prime}(y)+y \phi^{\prime}(y)-\lambda \phi(y)=0
$$

which, in the complex $y$-plane cut from -1 to $-\infty$, has as linearly-independent solutions the Legendre functions $P_{\nu}(y)$ and $Q_{\nu}(y)$ with $\nu(\nu+1)=2 \lambda$. For later use in the boundaryvalue problem, we note that on the real interval $y \in(-1,0] \bar{u}^{(0)}$ and $\bar{w}^{(0)}$ can be expressed as the linear combinations

$$
\begin{aligned}
\bar{u}_{0}^{(0)} & =\alpha_{0}(\nu) P_{\nu}(y)+\beta_{0}(\nu) Q_{\nu}(y), \\
\bar{w}^{(0)} & =\alpha(\nu) P_{\nu}(y)+\beta(\nu) Q_{\nu}(y)
\end{aligned}
$$

with

$$
\begin{aligned}
\alpha_{0}(\nu) & =\cos (\nu \pi), \quad \beta_{0}(\nu)=-\frac{2}{\pi} \sin (\nu \pi), \\
\alpha(\nu) & =[\log 2-\psi(\nu+1)] \alpha_{0}(\nu)-\frac{1}{2} \pi^{2} \beta_{0}(\nu), \\
\beta(\nu) & =2 \alpha_{0}(\nu)+[\log 2-\psi(\nu+1)] \beta_{0}(\nu)
\end{aligned}
$$

where $\psi(z)=\Gamma^{\prime}(z) / \Gamma(z)$ is the digamma function.

The expansions $\bar{v}_{k}(\eta)$. To obtain the outer expansions $\bar{v}_{k}$ which are recessive in the sectors $S_{k}$, we will use the WKBJ technique. Letting

$$
\bar{\chi}(\eta)=\exp \left\{\int^{\eta} g(\eta) d \eta\right\}
$$

and expanding $g(\eta)$ in the form

$$
g(\eta)=\epsilon^{-3 / 2} g_{0}(\eta)+g_{1}(\eta)+\epsilon^{3 / 2} g_{2}(\eta)+\cdots,
$$

we obtain a sequence of equations for $g_{n}(\eta)$ beginning with

and

$$
g_{0}{ }^{4}=\eta g_{0}{ }^{2}
$$

$$
\left(4 g_{0}{ }^{3}-2 \eta g_{0}\right) g_{1}=-6 g_{0}{ }^{2}\left(g_{0}{ }^{\prime}+\gamma g_{0}\right)+g_{0}(1+3 \gamma \eta)+\eta g_{0}{ }^{\prime} .
$$

Eq. (4.15) gives $g_{0}(\eta)=0$ or $g_{0}(\eta)= \pm \eta^{1 / 2}$. Since the trivial solution would lend to an expansion of balanced type, we must have $g_{0}(\eta)= \pm \eta^{1 / 2}$ and hence

$$
g_{1}(\eta)=-\frac{5}{2} \frac{g_{0}{ }^{\prime}}{g_{0}}-\frac{3}{2} \gamma(\eta)+\frac{1}{2} g_{0}^{-2}
$$

The transformation (4.13) now gives the WKBJ approximations

$$
\bar{v}_{ \pm}=\frac{1}{2} \pi^{-1 / 2} \epsilon^{3 / 4}\left(\eta \eta^{2}\right)^{-3 / 4} \exp \left\{ \pm \frac{2}{3} \epsilon^{-3 / 2} \eta^{3 / 2}\right\} \cdot G_{ \pm}\left(\eta, \epsilon^{3 / 2}\right)
$$

where $G_{ \pm}\left(\eta, \epsilon^{3 / 2}\right)$ is the Poincaré series

$$
G_{ \pm}\left(\eta, \epsilon^{3 / 2}\right)=1+\sum_{n=1}^{\infty}( \pm 1)^{n} \epsilon^{3 n / 2} G_{n}(\eta) .
$$


The normalization factor $\frac{1}{2} \pi^{-1 / 2} \epsilon^{3 / 4}$ in (4.17) has again been chosen so as to facilitate later matching to the inner expansions, and the eigenvalue $\lambda$ first appears in (4.18) at order $\epsilon^{3 / 2}$. To fix the branch in these expressions, it is convenient to place a cut along the Stokes line between $T_{1}$ and $T_{2}$ so that ph $\eta$ is in the range

$$
-4 \pi / 3<\operatorname{ph} \eta<2 \pi / 3 \text {. }
$$

In the complete sense, a given exact solution $V_{k}$ of (2.4) can be asymptotic to $\bar{v}_{+}$or $\bar{v}_{-}$ only in a sector of angle $2 \pi / 3$ bounded by Stokes lines. Accordingly, we define the three outer expansions $\bar{v}_{k}$ by the relations

$$
\begin{array}{ll}
\tilde{v}_{1}=-\bar{v}_{-}, & \eta \in T_{2} \cup T_{3}, \\
\bar{v}_{2}=i \bar{v}_{+}, & \eta \in T_{1} \cup T_{3}, \\
\bar{v}_{3}=-i \bar{v}_{+}, & \eta \in T_{2} .
\end{array}
$$

On crossing the branch cut from $T_{2}$ into $T_{1}$, we again note that the form of $\bar{v}_{3}$ will change but its value must remain the same. Since, beginning with $G_{2}(\eta)$, the expansions about $\eta=0$ of coefficients in (4.18) may contain logarithmic terms, we must therefore have

$$
\bar{v}_{3}=\bar{v}_{-}\left\{1+O\left(\epsilon^{3}\right)\right\} \quad \text { for } \quad \eta \in T_{1} .
$$

The expansions $\bar{u}_{k}$ and $\bar{v}_{k}$ are not, of course, valid at the turning point itself. However, they remain valid asymptotic representations for solutions of Eq. (2.4) down to $|\eta|$ greater than order $\epsilon$.

5. The central matching problem and composite expansions. To solve the central matching problem, one wishes to relate inner and outer expansions so that they represent different asymptotic approximations to the same solutions. For our case, normalizations will be fixed by assuming that the outer expansions of the previous section are asymptotic to the exact solutions of Eq. (2.4). Thus, when $|\eta|$ is larger than order $\epsilon$, we have

$$
U_{0}(\eta) \sim \bar{u}_{0}(\eta), \quad U_{k}(\eta) \sim \bar{u}_{k}(\eta), \quad V_{k}(\eta) \sim \bar{v}_{k}(\eta) .
$$

Close to the turning, the exact solutions must now be asymptotic to the combinations of inner expansions

$$
\begin{aligned}
U_{0}(\eta) & \sim B_{0}(\epsilon) \tilde{u}_{0}(\xi), \\
U_{k}(\eta) & \sim C_{k}(\epsilon) \tilde{u}_{k}(\xi)+B_{k}(\epsilon) \tilde{u}_{0}(\xi), \\
V_{k}(\eta) & \sim D_{k}(\epsilon) \tilde{v}_{k}(\xi)
\end{aligned}
$$

where the quantities $B_{0}, B_{k}, C_{k}$, and $D_{k}$ above (which depend only on $\epsilon$ ) are known as the central matching coefficients. With our normalizations, the convergent series $\bar{u}_{0}$ and $\tilde{u}_{0}$ are simply different representations of $U_{0}$ and hence $B_{0}(\epsilon) \equiv 1$. To obtain approximations to the remaining matching coefficients, we will use inner and outer expansion operators and the asymptotic matching principle [3].

Let $f(\eta, \epsilon)$ be a given function. Then, if $E_{p}$ denotes the outer expansion operator, $E_{p} f$ is the partial sum of the outer expansion for $f$ up to and including terms of order $\epsilon^{p}$. Similarly, if $H_{a}$ denotes the inner expansion operator, $H_{a} f$ is the inner expansion for $f$ up to and including terms of order $\epsilon^{a}$. For $\eta$ in the overlap region $\mathcal{O}$ where both inner and 
outer expansions are valid, the asymptotic matching principle may now be stated in the form

$$
\left(E_{p} H_{a}-H_{a} E_{p}\right) f=0 .
$$

To obtain approximations to the matching coefficients associated with the solutions $U_{k}(\eta)$, the matching must be performed with $\eta$ in the sector $T_{k}$ where both $\bar{u}_{k}$ and $\tilde{u}_{k}$ (for $|\xi| \rightarrow \infty$ ) are purely balanced. When $p=0$ and $q=1$, for $\eta \in T_{k} \cap \mathcal{O}$ we have

$$
\begin{aligned}
H_{1} E_{0} U_{k} & =H_{1} \bar{u}_{k}{ }^{(0)}(\epsilon \xi) \\
& =\gamma+\left[\frac{3}{5}+\lambda(2-\gamma)\right] \epsilon \xi+[1-\lambda \epsilon \xi] \log \epsilon \xi
\end{aligned}
$$

where $\gamma$ is the Euler constant, while

$$
E_{0} H_{1} U_{k}=E_{0} H_{1}\left[C_{k}(\epsilon) \tilde{u}_{k}(\xi)+B_{k}(\epsilon) \tilde{u}_{0}(\xi)\right] .
$$

These expressions suggest that $B_{k}(\epsilon)$ and $C_{k}(\epsilon)$ have expansions with respect to the asymptotic sequence $\log \epsilon, 1, \epsilon \log \epsilon, \epsilon, \epsilon^{2} \log ^{2} \epsilon, \epsilon^{2} \log \epsilon, \epsilon^{2}, \cdots$. The matching principle with $p=3$ and $q=4$ now gives the approximations

$$
B_{k}(\epsilon)=\log \epsilon+O\left(\epsilon^{3} \log ^{2} \epsilon\right)
$$

and

$$
C_{k}(\epsilon)=-1+O\left(\epsilon^{3} \log \epsilon\right) .
$$

For the solutions $V_{k}(\eta)$, the matching must be performed in the sectors $S_{k}$ where $\bar{v}_{k}$ and $\tilde{v}_{k}$ (as $\left.|\xi| \rightarrow+\infty\right)$ are purely recessive. However, because of the rapidly varying exponential factors, the operator $E_{p}$ cannot be applied directly. Rather, following Reid [9], we define the "modified" outer expansion operators

$$
E_{p}{ }^{ \pm}=G^{ \pm} E_{p} G^{\mp}
$$

where

$$
\begin{aligned}
G^{ \pm} & =\frac{1}{2} \pi^{-1 / 2} \epsilon^{3 / 4} \eta^{-3 / 4} \exp \left\{ \pm \frac{2}{3} \epsilon^{-3 / 2} \eta^{3 / 2}\right\} \\
& =\frac{1}{2} \pi^{-1 / 2} \xi^{-3 / 4} \exp \left\{ \pm \frac{2}{3} \xi^{3 / 2}\right\},
\end{aligned}
$$

and for $\eta \in S_{k} \cap \mathcal{O}$ use the matching principle

$$
\left(E_{p}^{ \pm} H_{a}-H_{a} E_{p}^{ \pm}\right) f=0 .
$$

A short calculation now shows

$$
D_{k}(\epsilon)=1+O\left(\epsilon^{3} \log \epsilon\right) .
$$

In addition to their uses in the matching procedure above, the expansion operators $E_{p}$ and $H_{a}$ can also be used to obtain composite expansions for the solutions of Eq. (2.4). In particular, we may define additive and multiplicative expansion operators $a_{p, e}$ and $\mathfrak{T}_{p, q}$, respectively, by the relations

$$
Q_{p, a} f=\left[E_{p}+H_{a}-E_{p} H_{q}\right] f
$$

and

$$
\mathfrak{M}_{p, a} f=\frac{\left(E_{p} f\right)\left(H_{q} f\right)}{E_{p} H_{a} f}
$$


The term $E_{p} H_{a} f$ (or, because of (5.3), $H_{a} E_{p} f$ ) here is the common part of both inner and outer expansions for $f$. Applying $E_{p}$ or $H_{q}$ to either of the above gives simply $E_{p} f$ or $H_{q} f$, respectively. The choice between the additive and multiplicative composite expansion is usually based on convenience. At present, there is no rigorous theory giving reasons for prefering (5.12) to (5.13) or conversely.

The outer expansion $\bar{u}_{0}(\eta)$ contains the inner expansion $\tilde{u}_{0}(\xi)$ and is therefore a composite expansion for the solution $U_{0}(\eta)$. This expansion is valid in the entire complex $\eta$-plane $I$.

To obtain composite approximations for the solutions $U_{k}(\eta)$, we will use the additive operator $a_{p, a}$. In particular, if $\gamma$ is again the Euler constant,

$$
\begin{array}{r}
a_{0,1} U_{k}=\bar{u}_{k}{ }^{(0)}(\eta)-B_{k}(\xi ; 1,1)-\gamma-\log \xi+\epsilon \lambda\left\{B_{k}(\xi ; 2,1)+\xi[\gamma-1+\log \xi]\right\} \\
+\frac{3}{2} \gamma_{0} \epsilon B_{k}(\xi ;-1,1) .
\end{array}
$$

This expression is valid in the fan-shaped region $T_{k} \cup N_{\epsilon}$ where $N_{\epsilon}$ denotes the small neighborhood of the turning point in which the inner expansions are valid (see Fig. 3).

To obtain composite expansions for the solutions $V_{k}(\eta)$, we will use the multiplicative operator $\mathfrak{M}_{p, q}$ with $E_{p}$ in (5.13) replaced by the appropriate modifier operator $E_{p}{ }^{+}$or $E_{p}{ }^{-}$. We find that

$$
\mathfrak{T}_{0,0} V_{k}=\left[\frac{2 \eta}{\left(1-y^{2}\right)^{1 / 2}}\right]^{3 / 4} A_{k}(\xi, 1) .
$$

This expression is valid in the region $\left(I-T_{k}\right) \cup N_{\epsilon}$. In additional sectors of the complex $\eta$-plane, the composite expansions for both $U_{k}$ and $V_{k}$ will have somewhat different forms involving the continuation of the relevant outer expansion across a Stokes line. Although we will not explicitly do so, these forms can be obtained from the solution of the lateral connection problem.

6. The lateral connection problem. Six of the seven outer expansions in Sec. 4 are valid in the complete sense only in restricted sectors of the complex $\eta$-plane which have Stokes lines as boundaries. The continuation of outer expansions across these bounding Stokes lines is called the lateral connection problem. A solution of the lateral
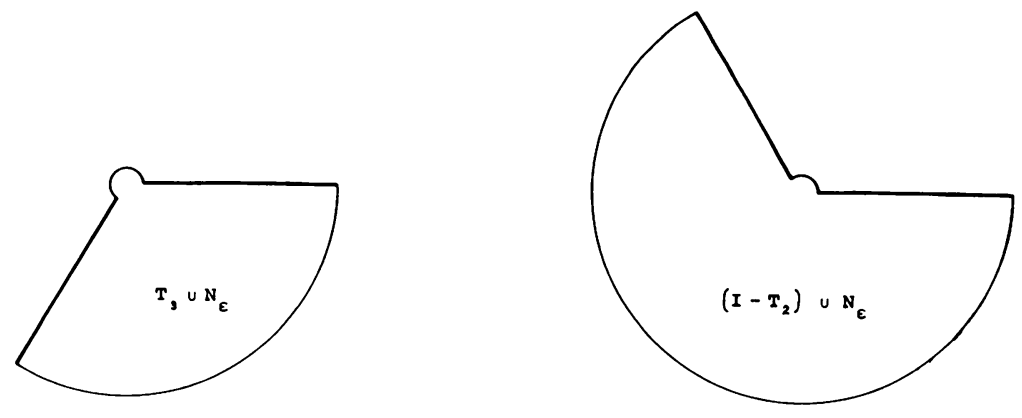

$n-p l a n e$

Fig. 3. Restricted domains of validity for the composite expansions $Q_{p, q} U_{3}(\eta)$ (left) and $\Re_{p, q} V_{2}(\eta)$ (right) in the $\eta$-plane. 
connection problem is, of course, included in a complete solution of the central matching problem (but not conversely). In this section, we will derive approximations to the Stokes multipliers which characterize the required continuations. This, in effect, corresponds to deriving approximations for the connection formulas which relate the seven outer expansions. If we knew the connection formulas for the exact solutions themselves, it would be a trivial matter to obtain the relevant Stokes multipliers. Unfortunately, these exact connection formulas are unknown. However, from the exact formulas relating the special functions in Sec. 3, we can obtain approximations involving the inner expansions. For example,

$$
\begin{aligned}
& H_{1} \tilde{u}_{3}-H_{1} \tilde{u}_{1}=2 \pi i H_{1} \tilde{v}_{2}, \\
& H_{1} \tilde{u}_{1}-H_{1} \tilde{u}_{2}=2 \pi i H_{1} \tilde{v}_{3},
\end{aligned}
$$

while

$$
H_{1} \tilde{u}_{2}-H_{1} \tilde{u}_{3}=2 \pi i\left[H_{1} \tilde{v}_{1}+H_{1} \tilde{u}_{0}\right] .
$$

As is consistent with (3.20), adding these gives the additional relation

$$
H_{1}\left(\tilde{v}_{1}+\tilde{v}_{2}+\tilde{v}_{3}\right)=-H_{1} \tilde{u}_{0}
$$

which is an approximation to a general relation of the form

$$
\sum_{i=1}^{3} \tilde{v}_{i}=\beta_{0}(\epsilon) \tilde{u}_{0}+\beta_{1}(\epsilon) \tilde{u}_{1} .
$$

Since $A_{k}(\xi ; 1,1)$ first appears in $\tilde{v}_{k}^{(3)}(\xi), \beta_{1}(\epsilon)$ above must be of order $\epsilon^{3}$. From the central matching problem, we know which combinations of the inner expansions match to each outer expansion in an appropriate sector of the complex plane. Hence, relations like (6.1) and (6.2) allow determination of the Stokes multipliers.

The outer expansion of $U_{0}(\eta)$ is valid in all of $I$. Of the other six solutions, we will consider explicitly only the two representatives $U_{3}(\eta)$ and $V_{2}(\eta)$. The outer expansion of $U_{3}(\eta)$ in the sector $T_{3}$ is $\bar{u}_{3}$ while the matched inner expansion is

$$
U_{3} \sim C_{3}(\epsilon) \tilde{u}_{3}(\xi)+B_{3}(\epsilon) \tilde{u}_{0}(\xi) .
$$

On crossing the Stokes line into $T_{1}$, the outer expansion of $U_{3}$ must discontinuously pick up a multiple of $\bar{v}_{2}$ which is purely recessive in $S_{2}$. Similarly, on crossing the Stokes line into $T_{2}$, the outer expansion of $U_{3}$ must discontinuously pick up multiples of both $\bar{v}_{1}$ and $\bar{u}_{0}$. Formulas like (6.1) show that

$$
\begin{aligned}
U_{3} & \sim \bar{u}_{3}, & & \eta \in T_{3}, \\
& \sim \bar{u}_{2}+s_{0} \bar{u}_{0}+s_{2} \bar{v}_{1}, & & \eta \in T_{2}, \\
& \sim \bar{u}_{1}+s_{1} \bar{v}_{2}, & & \eta \in T_{1},
\end{aligned}
$$

where $\bar{u}_{0}, \bar{u}_{1}, \bar{v}_{1}$, etc. denote the expressions in Sec. 4 and the Stokes multipliers are (with our normalizations)

$$
s_{0}=-s_{1}=s_{2}=2 \pi i\left[1+O\left(\epsilon^{3} \log \epsilon\right)\right] .
$$

There are similar expressions for $U_{1}$ and $U_{2}$. However, for $U_{2}$ the multiple of $\bar{u}_{0}$ is present in the sector $T_{3}$ rather than $T_{2}$ and it is entirely absent in $U_{1}$. 
The outer expansion of $V_{2}(\eta)$ in the sector $T_{1} \cup T_{3}$ is $\bar{v}_{2}$ while the matched inner expansion is

$$
V_{2} \sim D_{2}(\epsilon) \bar{v}_{2}(\epsilon)
$$

Relation (6.3) implies that

$$
\begin{aligned}
V_{2} & \sim \bar{v}_{2}, & & \eta \in T_{1} \cup T_{3}, \\
& \sim-\bar{v}_{3}+s_{3} \bar{v}_{1}+s_{4} \bar{u}_{0}+s_{5} \bar{u}_{1}, & & \eta \in T_{2},
\end{aligned}
$$

where the Stokes multipliers for this solution are

$$
s_{3}=s_{4}=-1+O\left(\epsilon^{3} \log \epsilon\right), \quad s_{5}=O\left(\epsilon^{3}\right) .
$$

Similar expressions hold for $V_{1}$ and $V_{3}$. We note that the outer expansion of $V_{1}$ is maximally recessive on the Stokes line $\mathrm{ph} \eta=0$.

The behavior of the outer expansions on the Stokes lines themselves is a somewhat delicate question which has not yet been rigorously answered. However, the only choice consistent with the exact connection formulas (3.19) and (3.20) is that the expansion valid on a Stokes line should be the mean of the complete expansions valid on either side of the Stokes line. Dominant terms in an expansion will thus be continuous across Stokes lines.

7. The boundary-value problem. We now wish to use the matched expansions of the previous sections to study the boundary-value problem (1.1) and (1.2) (transformed into the $\eta$ variable). A fundamental set of linearly independent solutions of Eq. (2.4) consists of $U_{0}(\eta)$, one of the three solutions $U_{k}(\eta)$, and two of the three solutions $V_{k}(\eta)$ $(k=1,2,3)$. We will choose the set

$$
\left\{U_{0}, U_{3}, V_{1}, V_{2}\right\}
$$

which is "numerically satisfactory" in the sense of Miller [7]. To express the boundary conditions (1.2) in terms of $\eta$, we let

$$
\eta_{1}=\eta(0)=\frac{1}{2}(3 \pi / 2)^{2 / 3}
$$

and denote a general solution of Eq. (2.4) by $\chi(\eta)$. The first two conditions in (1.2) now become

$$
\chi\left(\eta_{1}\right)=\chi^{\prime}\left(\eta_{1}\right)=0 .
$$

Conditions at the turning point $\eta=0$ are slightly more complicated. If the differential operators $B_{2}$ and $B_{3}$ are defined by

$$
\begin{aligned}
& \AA_{2}=\frac{d^{2}}{d \eta^{2}}+\gamma(\eta) \frac{d}{d \eta}, \\
& \AA_{3}=\frac{d^{3}}{d \eta^{3}}+\left[\gamma^{\prime}(\eta)+\gamma^{2}(\eta)\right] \frac{d}{d \eta},
\end{aligned}
$$

then the second two conditions in (1.2) become

$$
\beta_{2} \chi(0)=B_{3} \chi(0)=0 .
$$


Since $\chi(\eta)$ must be a linear combination of the solutions in the set (7.1), conditions (7.2) and (7.4) give a characteristic equation involving a four-by-four determinant. In particular, we have



In this equation there are three distinct types of terms. We may write (7.5) as

$$
D+B+R=0
$$

where terms in $D$ involve a multiple of either $V_{2}\left(\eta_{1}\right)$ or $V_{2}{ }^{\prime}\left(\eta_{1}\right)$ and are dominant while terms in $B$ and $R$ are balanced and recessive, respectively. Hence, for small $\epsilon$,

$$
D \gg B \gg R \text {. }
$$

Using the matched inner expansions at the turning point and outer expansions at $\eta_{1}$, we may write $D$ as

$$
\begin{aligned}
\mathscr{D} / D_{1}(\epsilon)=C_{3}(\epsilon) & W\left(\bar{u}_{0}, \bar{v}_{2}\right)\left(\eta_{1}\right) \mathfrak{N}\left(\tilde{v}_{1}, \tilde{u}_{3}\right)(0) \\
& -\left[W\left(\bar{u}_{3}, \bar{v}_{2}\right)\left(\eta_{1}\right)+\left\{\frac{1}{2} s_{0}-B_{3}(\epsilon)\right\} W\left(\bar{u}_{0}, \bar{v}_{2}\right)\left(\eta_{1}\right)\right] \Re\left(\tilde{v}_{1}, \tilde{u}_{0}\right)(0)
\end{aligned}
$$

where $s_{0}$ is the Stokes multiplier in (6.4),

$$
W(\bar{f}, \bar{g})(\eta)=\bar{f}(\eta) \bar{g}^{\prime}(\eta)-\bar{f}^{\prime}(\eta) \bar{g}(\eta)
$$

is a Wronskian, and

$$
\mathfrak{N}(\tilde{f}, \tilde{g})(\xi)=\bigotimes_{2} \tilde{f}(\xi) \mathbb{B}_{3} \tilde{g}(\xi)-\bigotimes_{3} \tilde{f}(\xi) \mathbb{B}_{2} \tilde{g}(\xi) .
$$

The characteristic equation (7.5) is quite complex and a complete analysis of the spectrum would involve extensive numerical computation. In the present work, our aim is to obtain analytically the asymptotic behaviour of the eigenvalue $\lambda(\epsilon)$ as $\epsilon \rightarrow 0$. Since this asymptote comes from the dominant terms in (7.5), we will therefore examine the approximate equation

$$
D=0 .
$$

Indeed, since the exponentials in both $D$ and $\mathscr{R}$ are maximally dominant and recessive, respectively, for ph $\eta=0$, by (7.7) Eq. (7.11) provides a consistent approximation to the full characteristic equation for values of $\epsilon$ only moderately small.

The first approximation to $D$ is

$$
\epsilon^{23 / 4} D=-\bar{v}_{2}{ }^{(0) \prime}\left(\eta_{1}\right) \bar{u}_{0}{ }^{(0)}\left(\eta_{1}\right)\left[\tilde{v}_{1}{ }^{(0) \prime \prime}(0) \tilde{u}_{3}{ }^{\left({ }^{\prime}\right)^{\prime \prime \prime}}(0)-\tilde{v}_{1}{ }^{(0) \prime \prime \prime}(0) \tilde{u}_{3}{ }^{(0) \prime \prime}(0)\right]+O(\epsilon) .
$$

Since $\eta=\eta_{1}$ corresponds to $y=0$, (7.11) and (4.11) now give that the first approximation to $\lambda$ comes from roots of the equation

$$
\cos (\nu \pi) P_{\nu}(0)-\frac{2}{\pi} \sin (\nu \pi) Q_{\nu}(0)=0
$$

with $\nu(\nu+1)=2 \lambda$. This implies $\nu$ must be an odd positive integer and hence, as $\epsilon \rightarrow 0$, 


$$
\lambda_{n}(\epsilon) \sim n(2 n-1) \quad n=1,2,3, \cdots .
$$

This result agrees with the lower bound obtained in [2] as the parameter $m l^{4} \Omega^{2} / E I$ gets large. Hence, this asymptote is approached from above.

\section{REFERENCES}

[1] R. Bisplinghoff, H. Ashley, and R. L. Halfman, Aeroelasticity, Addison-Wesley, Reading, Mass., 1955

[2] W. E. Boyce, R. C. Di Prima, G. H. Handelman, Vibrations of rotating beams of constant section, Second U. S. Nat. Congress Appl. Mech., 165-173 (1956)

[3] L. E. Fraenkel, On the method of matched asymptotic expansions, Parts I-III, Proc. Camb. Phil. Soc. 65, 209-284 (1969)

[4] P. C. Hughes and J. C. Fung, Liapunov stability of spinning satellites with long, flexible appendages, Celestial Mech. 4, 295-308 (1971)

[5] W. D. Lakin and W. H. Reid, Stokes multipliers for the Orr-Sommerfeld equation, Phil. Trans. Roy. Soc. Lond. A268, 325-349 (1970)

[6] H. Lo and J. Renbarger, Bending vibration of a rotating beam, First U. S. Nat. Congress Appl. Mech., 75-79 (1952)

[7] J. C. P. Miller, On the choice of standard solutions for a homogeneous linear differential equation of the second order, Quart. J. Mech. Appl. Math. 3, 225-235 (1950)

[8] F. W. J. Olver, in Asymptotic solutions of differential equations (C. H. Wilcox, ed.), John Wiley and Sons, New York, 1964

[9] W. H. Reid, Composite approximations to the solutions of the Orr-Sommerfeld equation, Studies in Appl. Math. 51, 341-368 (1972) 